\title{
MALARIA EN PACIENTES CON INFECCIÓN POR VIH: SERIE DE CASOS EN UN HOSPITAL PERUANO
}

\author{
Juan José Montenegro-Idrogo ${ }^{1,2, a}$, Renzo Vargas-Gonzales ${ }^{3, a}$, Moisés Sihuincha ${ }^{4, a}$
}

\begin{abstract}
RESUMEN
Las manifestaciones clínicas de la malaria en pacientes con VIH pueden ser variables dependiendo del estado inmunológico del paciente. La evidencia en relación a la coinfección es escasa a nivel nacional. Se describen cuatro casos procedentes de un hospital de Iquitos-Perú con diagnóstico de infección por VIH y examen de sangre positivo para malaria. De estos pacientes, dos tenían infección por Plasmodium falciparum y dos por Plasmodium vivax. Un paciente se encontraba en estadio sida con mala adherencia a la terapia antirretroviral combinada (TARVc) y el resto se encontraba en estadios tempranos sin recibir TARVc.
\end{abstract}

Palabras clave: Malaria; VIH; Paludismo; Coinfección (fuente: DeCS BIREME).

\section{MALARIA IN HIV-INFECTED PATIENTS: A SERIES OF CASES IN A PERUVIAN HOSPITAL}

\begin{abstract}
The clinical signs of malaria in HIV patients may vary depending on the immunological status of the patient. Nationally, evidence regarding co-infection is scarce. This research describes four cases from a hospital in lquitos, Peru, of patients diagnosed with HIV infection and a positive blood test for malaria. Two of these patients had Plasmodium falciparum infection, and two had Plasmodium vivax infection. One of the patients was in the AIDS stage with poor adherence to combination antiretroviral therapy (CART), and the other three were in the early stages and not receiving cART.
\end{abstract}

Keywords: Malaria; HIV; Paludisme; Coinfection (source: MeSH NLM).

\section{INTRODUCCIÓN}

La presentación clínica de malaria en pacientes con VIH puede ser variable dependiendo del estado inmunológico del paciente ${ }^{(1-4)}$. En regiones de África, donde las prevalencias de malaria e infección por VIH suele estar entre 10 a $20 \%$ en promedio ${ }^{(4-6)}$, la profilaxis con cotrimoxazol puede prevenir la malaria ${ }^{(4,5)}$. La manifestación más evidente de esta coinfección es la anemia ${ }^{(7,8)}$. La coinfección VIH y malaria es diferente en zonas de alta y baja endemicidad, siendo en las primeras más frecuente coinfección por Plasmodium falciparum ${ }^{(5,9,10)}$. Existe controversia por los resultados de diferentes estudios en relación al estado inmunológico ${ }^{(3,11)}$, profilaxis con antimaláricos ${ }^{(4,5,11)}$, uso de terapia antirretroviral combinada (TARVc) ${ }^{(12)}$ y la presentación clínica de esta coinfección; además, de su severidad y mortalidad ${ }^{(2,3,7,9)}$.

El Perú es considerado como una zona de baja endemicidad para la transmisión de la malaria y no existe evidencia previa de esta coinfección. En la Amazonía peruana (región Loreto), las provincias El Tigre, Alto Nanay y el Pastaza son consideradas de alto riesgo para transmisión de malaria por Plasmodium vivax (P. vivax) y por Plasmodium falciparum ${ }^{(P .}$ falciparum $)^{(13)}$.

El presente estudio describe cuatro casos de pacientes con malaria e infección con $\mathrm{VIH}$ atendidos en un hospital de una zona de baja endemicidad para malaria en lquitos, Loreto, Perú.

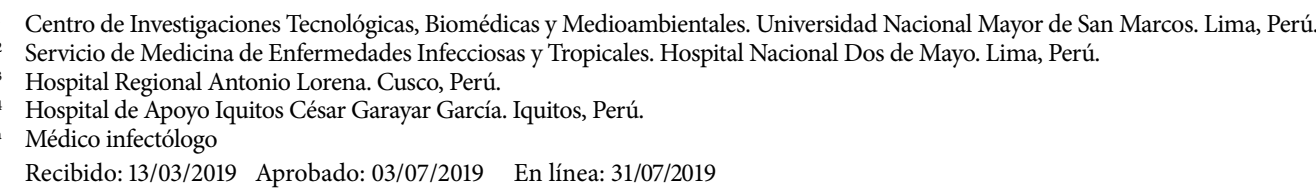

Citar como: Montenegro-Idrogo JJ, Vargas-Gonzales R, Sihuincha M. Malaria en pacientes con infección por VIH: serie de casos en un hospital peruano. Rev Peru Med Exp Salud Publica. 2019;36(3):520-4. doi:http://dx.doi.org/10.17843/rpmesp.2019.363.4370. 


\section{REPORTE DE CASOS}

Se realizó una búsqueda retrospectiva de los registros de pacientes con coinfección de malaria y VIH en un hospital peruano de una zona de baja endemicidad de malaria en lquitos, Perú, durante el periodo 2011-2016, que fueron atendidos por presentar síndrome febril. Encontramos cuatro pacientes que presentaron diagnóstico de infección por VIH y resultado positivo para malaria por examen de sangre periférica (gota gruesa).

Todos los pacientes eran adultos, la mayoría (3/4) estaban en estadio no sida, nunca se habían tratado con antirretrovirales (naive) y procedían o presentaban estancia en zona de transmisión de malaria. Sólo uno recibía TARVc con mala adherencia y en estadio sida. De los pacientes naive, dos presentaron infección por $\mathrm{VIH}$ como debut de la enfermedad, estos fueron positivos para $P$. vivax (uno con compromiso del sistema nervioso central con probable malaria cerebral) (Tabla 1).

Dos pacientes recibieron terapia antimalárica endovenosa (Caso 2 y 3) con artesunato y clindamicina por malaria grave, un paciente con infección por $P$. falciparum no complicada recibió artesunato más mefloquina y el paciente con infección por $P$. vivax no complicada recibió cloroquina más primaquina por vía oral.

Sólo el paciente con malaria por $P$. falciparum y en estadio C (Caso 2) presentó alta densidad parasitaria con negativización de las formas asexuadas en el tercer día y de las formas sexuadas en el sexto día en el examen de gota gruesa posterior a la terapia antiparasitaria. El resto de los pacientes obtuvieron negativización de las formas asexuadas y sexuadas en la primera muestra control posterior al día de inicio de la terapia antimalárica. El otro paciente con infección por $P$. falciparum (Caso 1) con hemoglobina de $9 \mathrm{gr} / \mathrm{dl}$ inició TARVc con zidovudina, lamivudina y efavirenz durante el episodio de malaria sin complicaciones posteriores en los controles mensuales.

\section{DISCUSIÓN}

La presente serie describe una baja frecuencia de presentación de malaria en pacientes con $\mathrm{VIH}$ reportados en un hospital que atiende pacientes procedentes de zonas de baja endemicidad para malaria. Las frecuencias de malaria y VIH varian dependiendo de la región.

Un metaanálisis que incluyó 23 estudios con elevada heterogeneidad y sesgo de publicación describió una prevalencia combinada de $19 \%$ de coinfección de malaria y $\mathrm{VIH}$, siendo mayor en adultos y menor en gestantes y niños ${ }^{(8)}$.
En Ghana, en 220 pacientes con malaria se reportó una prevalencia de coinfección con VIH de 15,5\%, todos con niveles de CD4 bajos ${ }^{(6)}$. En 761 pacientes infectados con VIH de un hospital en Nigeria la prevalencia de coinfección con malaria fue $27,7 \%$ y cerca de la totalidad por $P$. falciparum ${ }^{(5)}$. En otro estudio realizado en Nigeria la frecuencia de malaria en personas no $\mathrm{VIH}$ fue de $25,8 \%$, mientras que en personas con $\mathrm{VIH}$ la frecuencia de coinfección fue cerca del $50 \%{ }^{(10)}$.

El Sureste asiático es considerada como una zona de baja endemicidad para la malaria; en la frontera entre Tailandia y Myanmar se examinaron a 867 personas encontrándose una prevalencia de 1,8\% para malaria y tasas de coinfección con $\mathrm{VIH}$ de $0,8 \%$ para $P$. falciparum y $1,0 \%$ para P. vivax ${ }^{(7)}$. En 858 personas de Gabón que fueron tamizadas para malaria la prevalencia de parasitemia asintomática fue de $13,5 \%$, menor en personas con $\mathrm{VIH}(7,1 \%)^{(4)}$. En una cohorte de 336 personas con malaria por $P$. falciparum en Soweto, Sudáfrica se encontró una coinfección con $\mathrm{VIH}$ de $33,0 \%$ y mayor desarrollo de malaria grave en pacientes con $\mathrm{VIH}$ no inmunes a malaria ${ }^{(2)}$. Sudamérica es considerada como zona de baja endemicidad para malaria y presenta poca evidencia de esta coinfección ${ }^{(14)}$, se menciona que la infección por VIH se relaciona con la severidad de la malaria dependiendo del estado inmunológico o estadio clínico del $\mathrm{VIH}^{(15)}$.

De los casos descritos, dos presentaron diagnóstico de $\mathrm{VIH}$ como debut del episodio de malaria (ambos por $P$. vivax); cabe mencionar que en ambos pacientes ninguno presentó estadio clínico tardío (sida) y los diagnósticos se realizaron por tamizaje y por descarte de presentación inusual de la enfermedad (paciente con malaria grave por compromiso neurológico). Los otros dos pacientes tenían diagnóstico previo de $\mathrm{VIH}$ (ambos presentaron malaria por $P$. falciparum), uno presentó diagnóstivo de $\mathrm{VIH}$ meses previos al episodio de malaria, el otro tenía el diagnóstivo de $\mathrm{VIH}$ un año previo con progresión a estadio sida, con estigmas de inmunosupresión severa, con mala adherencia a la TARVc y con mayor demora en el aclaramiento parasitológico de las muestras de sangre. Ninguno de ellos recibió profilaxis con cotrimoxazol.

Se menciona que el estado inmunológico predispone a una mayor coinfección de malaria y VIH; así, niveles bajos de CD4 se relacionan a mayor presentación de malaria ${ }^{(2,4,6)}$; además, la coinfección de malaria y $\mathrm{VIH}$ puede relacionarse a una disminución de los niveles de CD4 ${ }^{(5)}$, lo que aumenta el riesgo y gravedad de la infección, la carga parasitaria y facilita la tasa de transmisión. La infección por malaria también se asocia con una fuerte activación de las células CD4+ y una regulación positiva de las citocinas proinflamatorias, proporcionando un microambiente ideal para la propagación del virus entre las células $\mathrm{CD} 4+\mathrm{y}$ una rápida replicación del $\mathrm{VIH}$, y con ello un aumento en la progresión de la enfermedad ${ }^{(3)}$. 
Tabla 1. Características demográficas, clínicas y de diagnóstico de los pacientes con coinfección malaria-VIH en un hospital de Iquitos. Iquitos, Perú

\begin{tabular}{|c|c|c|c|c|}
\hline Características & Caso 1 & Caso 2 & Caso 3 & Caso 4 \\
\hline Edad (años) & 55 & 30 & 35 & 43 \\
\hline Sexo & Masculino & Masculino & Femenino & Masculino \\
\hline Procedencia & lquitos & Punchana, Iquitos & Iquitos & Iquitos \\
\hline Historia de viajes & $\begin{array}{l}\text { Putumayo, Yaraví, } \\
\text { Caballococha (Loreto) }\end{array}$ & No & No & No \\
\hline Trabajo & Policía & Leñador & Labor doméstica & Desconocido \\
\hline Tiempo diagnóstico VIH (meses) & 12 & 5 & $\begin{array}{l}\text { Episodio actual } \\
\text { (debut) }\end{array}$ & $\begin{array}{l}\text { Episodio actual } \\
\text { (debut) }\end{array}$ \\
\hline CD4 (células/mm³) & 204 & 80 & 350 & - \\
\hline Carga viral (copias/mm³) & 3203967 & 346141 & 105000 & - \\
\hline Estadio clínico (OMS) & B & C & $A$ & A \\
\hline Historia previa de TARVc & No & $\mathrm{AZT}+3 \mathrm{TC}+\mathrm{EF}$ (No adherente) & No & No \\
\hline Profilaxis & No & No & No & No \\
\hline Historia previa de malaria & No & No & No & No \\
\hline $\begin{array}{l}\text { Antecedentes médicos } \\
\text { (incluye tuberculosis) }\end{array}$ & No & No & No & No \\
\hline \multicolumn{5}{|l|}{ Características clínicas } \\
\hline Inicio de síntomas (días) & 11 & 6 & 6 & 7 \\
\hline Manifestaciones generales & Fiebre + escalofríos & Fiebre + diaforesis & Fiebre + escalofríos & Fiebre + diaforesis \\
\hline Síntomas respiratorios & No & Disnea & No & No \\
\hline Síntomas neurológicos & Cefalea & No & $\begin{array}{l}\text { Cefalea + trastorno de } \\
\text { sensorio } \\
\text { Convulsiones+ Glasgow 6/15 }\end{array}$ & Cefalea leve \\
\hline Síntomas abdominales & $\begin{array}{l}\text { Dolor epigastrio, } \\
\text { hepato-esplenomegalia }\end{array}$ & Diarrea crónica & $\begin{array}{l}\text { Dolor epigastrio, } \\
\text { diarrea aguda }\end{array}$ & $\begin{array}{l}\text { Dolor abdominal, } \\
\text { diarrea aguda }\end{array}$ \\
\hline \multicolumn{5}{|l|}{ Exámenes de laboratorio } \\
\hline \multicolumn{5}{|l|}{ Hemograma } \\
\hline $\begin{array}{l}\text { Hemoglobina }(\mathrm{g} / \mathrm{dL}) / \\
\text { Hematocrito }(\%)\end{array}$ & $9,0 / 26,3$ & $-/ 15,0$ & $10,5 / 33,0$ & $14,1 /-$ \\
\hline Leucocitos (A, S, L) & $2900(0,35,56)$ & $5300(4,62,30)$ & $7720(0,43,40)$ & $4670(-)$ \\
\hline Plaquetas (unidades/ml) & 133000 & 419000 & 176000 & 67000 \\
\hline Tiempo Protrombina (s) & 16 & 12 & - & - \\
\hline \multicolumn{5}{|l|}{ Perfil Hepático } \\
\hline Albúmina & 2,4 & 2,8 & 3,9 & - \\
\hline Transaminasas AST/ALT & $91 / 56$ & 9/7 & - & - \\
\hline Bilirrubina total (mg/dL) & 0,36 & 0,45 & - & - \\
\hline Bilirrubina directa $(\mathrm{mg} / \mathrm{dL})$ & 0,16 & 0,20 & - & - \\
\hline \multicolumn{5}{|l|}{ Perfil Renal } \\
\hline Creatinina $(\mathrm{mg} / \mathrm{dL})$ & 1,0 & 1,1 & 0,89 & 0,87 \\
\hline Urea $(\mathrm{mg} / \mathrm{dL})$ & 21,0 & 33,0 & - & - \\
\hline Complicaciones condicionantes & No & No & Sistema nervioso central & No \\
\hline Diagnóstico de malaria & $\begin{array}{l}\text { P. falciparum (++) (Quinto día de } \\
\text { hospitalización) }\end{array}$ & $\begin{array}{l}\text { P. falciparum (+++) (Primer día } \\
\text { de hospitalización) }\end{array}$ & $\begin{array}{l}\text { P. vivax (++) (Dos días antes } \\
\text { de la admisión) }\end{array}$ & $\begin{array}{l}\text { P. vivax (+) (En la } \\
\quad \text { admisión) }\end{array}$ \\
\hline Tratamiento & $\begin{array}{c}\text { Artesunato+ mefloquina } \\
\text { SMX -TMP + clindamicina } \\
\text { Ceftriaxona } \\
\text { Transfusión de eritrocitos }\end{array}$ & $\begin{array}{l}\text { Artesunato+clindamicina } \\
\text { Ceftriaxona } \\
\text { Transfusión de eritrocitos }\end{array}$ & $\begin{array}{l}\text { Artesunato+clindamicina } \\
\text { Ceftriaxona }\end{array}$ & $\begin{array}{l}\text { Cloroquina + } \\
\text { primaquina }\end{array}$ \\
\hline Inicio TARVc & $\begin{array}{c}\text { AZT+3TC+EFV } \\
\text { (Sexto día hospitalización) }\end{array}$ & Previo a la hospitalización & No en hospitalización & No en hospitalización \\
\hline Evolución Parasitológica & Negativo en muestras control & $\begin{array}{l}\text { Segundo día tratamiento: } \mathrm{F} \\
\qquad(+++) \\
\text { Cuarto y quinto día: } \mathrm{Fg}(15 \text { y } 5) \\
\text { Sexto a décimo día: negativo }\end{array}$ & Negativo en muestras control & $\begin{array}{l}\text { Negativo en } \\
\text { muestras control }\end{array}$ \\
\hline Evolución clínica & $\begin{array}{l}\text { Afebril y alta el día } 14 \text { luego de } \\
\text { hospitalización }\end{array}$ & $\begin{array}{l}\text { Afebril y alta el día } 11 \text { luego de } \\
\text { hospitalización }\end{array}$ & $\begin{array}{c}\text { Mejoría neurológica } \\
\text { (día 3) } \\
\text { Afebril y alta el día } 7 \text { luego de } \\
\text { hospitalización }\end{array}$ & $\begin{array}{l}\text { Alta el día } 2 \text { luego de } \\
\text { hospitalización }\end{array}$ \\
\hline \multicolumn{5}{|l|}{ Serología } \\
\hline Dengue & - & - & $\operatorname{lgM}(-)$ & $\operatorname{lgM}(-)$ \\
\hline Hepatitis B & NR & - & - & - \\
\hline Leptospirosis & $\lg M(-) / \lg G(-)$ & - & $\lg \mathrm{M}(-)$ & IgM Indeterminado \\
\hline Otros & No & No & $\begin{array}{c}\text { Raquicentesis: pleocitosis } \\
\text { mononuclear, proteinorraquia* }\end{array}$ & RPR: NR \\
\hline
\end{tabular}

A: Abastonados, S: segmentados, L: linfocitos, TARVc: Terapia antirretroviral combinada, F: formas asexuadas, Fg: formas gametocíticas, AZT: zidovudina, 3TC: lamivudina, EFV: efavirenz; RPR: serología sifilis, SMX-TMP: sulfametoxazol-trimetoprima (cotrimoxazol), NR: no reactivo

* Líquido cefalorraquídeo: leucocitos: 22 células $/ \mathrm{mm}^{3}$ por campo (L: $70 \%$ ), proteínas: $163 \mathrm{md} / \mathrm{dL}$, glucosa: $63 \mathrm{mg} / \mathrm{dL}$, gram, Ziehl Neelsen y tinta china negativos, guión: dato no disponible 
Algunos estudios refieren que el uso de TARVc evidencia menores tasas de infección por malaria en pacientes con $\mathrm{VIH}{ }^{(5,12)}$, en nuestra serie los pacientes con diagnóstico previo de VIH (ambos con malaria por $P$. falciparum) recibieron TARVc en el episodio actual de malaria (El caso 2 refería mala adherencia y no control virológico con estadio clínico C, el caso 1 inició al sexto día de hospitalización). El caso 2 evidenció retraso en aclaramiento parasitológico en relación a los otros casos, y el caso 1 no presentó mayores complicaciones, posterior al inicio de TARVc. En ambos, el esquema que recibieron fue zidovudina, lamivudina y efavirenz.

El uso de la profilaxis es discutible, se ha evidenciado que en regiones endémicas la profilaxis con cotrimoxazol reduce la presentación e incidencia de malaria en personas con VIH ${ }^{(4,5)}$. Un subanálisis del estudio COSTOP en adultos ugandeses con $\mathrm{VIH}$ con CD4 $\geq 250$ células/ $\mu$ y que recibían TARVc evaluó grupos para continuar o suspender la profilaxis con cotrimoxazol se evidenció que la incidencia de malaria fue de cuatro episodios/100 años-persona en los tratados con cotrimoxazol y 14 episodios/100 años-persona en tratados con placebo ${ }^{(1)}$. En nuestra serie, ningún paciente recibió profilaxis previa con cotrimoxazol, pero en el manejo hospitalario todos reportaron su uso, inclusive al alta. En el seguimiento ambulatorio no se evidenció recidiva a los seis meses. Una revisión en gestantes con VIH no encontró inferioridad en el riesgo de malaria placentaria en los grupos que usaron profilaxis con cotrimoxazol solo, en relación con aquellas que usaron, además, mefloquina; en estos últimos se evidenció una reducción de la carga parasitaria placentaria sin diferencia en la incidencia o complicaciones. No obstante, la evidencia es limitada y los efectos de la administración son mayores en el grupo que utilizó mefloquina ${ }^{(11)}$
Las características de mayor severidad son la anemia ${ }^{(6,7,10)}$ y la alta carga parasitaria ${ }^{(4,7)}$ dependiente del estado inmunológico o de la condición de $\mathrm{VIH}$ que es usualmente descrita; en nuestra serie el paciente en estadio clínico $\mathrm{C}$ evidenció dos días de demora en el aclaramiento parasitario de formas asexuadas, además, de la mayor severidad de anemia; a diferencia de los demás pacientes cuyos valores de anemia fueron moderados con aclaramiento parasitario en el primer control de primer día postratamiento.

Esta es la primera serie de casos de esta coinfección en una zona de baja endemicidad de malaria de la Amazonía peruana. Es posible la existencia de un subregistro de casos de coinfección VIH-malaria, ya que usualmente en pacientes con malaria no se realiza tamizaje para $\mathrm{VIH}$. La presentación en la mayoría de los pacientes fue en estadios no sida, el estado de inmunosupresión puede presentarse con severidad del cuadro de malaria y con prolongación del tiempo de aclaramiento parasitémico posterior al tratamiento. No se describe preferencia según el tipo de Plasmodium; sin embargo, $P$. falciparum se presentó en pacientes con diagnóstico previo de $\mathrm{VIH}$ y $P$. vivax se presentó en pacientes cuyo debut de la enfermedad fue por $\mathrm{VIH}$.

Criterios éticos: No se aplicó el consentimiento informado por ser una serie de casos retrospectiva. El estudio cuenta con el permiso del servicio del hospital donde se realizó el estudio.

Contribuciones de autoría: JMI, MSM y RVG han participado en la concepción del artículo, la recolección de datos, su redacción y aprobación de la versión final.

Fuentes de financiamiento: ninguno.

Conflicto de interés: ninguno.

\section{REFERENCIAS BIBLIOGRÁFICAS}

1. Kasirye R, Grosskurth H, Munderi P, Levin J, Anywaine Z, Nunn A, et al. Longitudinal effect of CD4 by cotrimoxazole use on malaria incidence among HIV-infected Ugandan adults on antiretroviral therapy: a randomized controlled study. Malar J. 2016;15:361. doi: 10.1186/s12936-016-1426-z.

2. Cohen C, Karstaedt A, Frean J, Thomas J, Govender N, Prentice E, et al. Increased prevalence of severe malaria in HIV-infected adults in South Africa. Clin Infect Dis. 2005;41(11):1631-7.

3. Alemu A, Shiferaw Y, Addis Z, Mathewos B, Birhan W. Effect of malaria on HIV/AIDS transmission and progression. Parasit Vectors. 2013;6:18. doi: 10.1186/1756-3305-6-18.
4. Bouyou Akotet MK, Koumba Lengongo JV, Ondounda M, Kendjo E, Mongo Delis A, Essomeyo Mebale M, et al. Burden of asymptomatic malaria, anemia and relationship with cotrimoxazole use and CD4 cell count among HIV1infected adults living in Gabon, Central Africa. Pathog Glob Health. 2017;2:1-9. doi:10.1080/20477724.2017.1401760.

5. Jegede FE, Oyeyi TI, Abdulrahman SA, Mbah HA, Badru T, Agbakwuru C, et al. Effect of HIV and malaria parasites co-infection on immune-hematological profiles among patients attending antiretroviral treatment (ART) clinic in Infectious Disease Hospital Kano, Nigeria. PLoS One. 2017;12(3):e0174233. doi: 10.1371/journal.pone.0174233.

6. Tagoe DN, Boachie J. Assessment of the impact of malaria on CD4+ T Cells and haemoglobin levels of HIV-malaria co-infected patients. J Infect Dev Ctries. 2012;6(9):660-63.

7. Rattanapunya $S$, Kuesap J, Chaijaroenkul W, Rueangweerayut R, Na-Bangchang K. Prevalence of malaria and HIV coinfection and influence of HIV infectionon malaria disease severity in population residing in malaria endemic area along the ThaiMyanmar border. Acta Trop. 2015;145:5560. doi: 10.1016/j.actatropica.2015.02.001.

8. Naing C, Sandhu NK, Wai VN. The Effect of Malaria and HIV Co-Infection on Anemia: A Meta-Analysis. Medicine (Baltimore). 2016;95(14):e3205. doi: 10.1097/MD.0000000000003205.

9. Rattanapunya S, Chaijaroenkul W, Kuesap J, Ruengweerayut R, NaBangchang K. Preliminary investigation on the prevalence of malaria and HIV co-infection in Mae Sot District, Tak 
Province of Thailand. Asian Pac J Trop Biomed. 20155(5):361-3.

10. Sanyaolu AO, Fagbenro-Beyioku AF, Oyibo WA, Badaru OS, Onyeabor OS, Nnaemeka CI. Malaria and HIV coinfection and their effect on haemoglobin levels from three health-care institutions in Lagos, southwest Nigeria. Afr Health Sci. 2013;13(2):295-300. doi: 10.4314/ahs. v13i2.14.

11. Denoeud-Ndam L, Zannou DM, Fourcade C, Taron-Brocard C, Porcher R, Atadokpede F, et al. Cotrimoxazole prophylaxis versus mefloquine intermittent preventive treatment to prevent malaria in HIV-infected pregnant women: two randomized controlled trials. J Acquir Immune Defic Syndr. 2014;65(2):198-206. doi: 10.1097/QAI.0000000000000058.

12. Kasirye RP, Grosskurth $H$, Munderi P, Levin J, Anywaine Z, Nunn A, et al. Effect of antiretroviral therapy on malaria incidence in HIV-infected Ugandan adults. AIDS.2017;31(4):577-582.

13. Ministerio de Salud del Perú, Centro Nacional de Epidemiología, Prevención y Control de Enfermedades. Caso de malaria 2013-2018 [Internet]. Lima: MINSA; 2018 [citado 02 de febrero de 2019]. Disponible en: http://www. dge.gob.pe/portal/docs/vigilancia/ sala/2108/SE03/malariapdf
14. Bastos FI, Barcellos C, Lowndes CM, Friedman SR. Co-infection with malaria and HIV in injecting drug users in Brazil: a new challenge to public health? Addiction. 1999; 94(8):1165-74.

15. Karp CL, Auwaerter PG. Co-infection with HIV and tropical infectious diseases. I. Protozoal pathogens. Clin Infect Dis. 2007;45(9):1208-13.

Correspondencia: Juan José Montenegro Idrogo Dirección: Av. Arnaldo Marquez 579 Int 507. Lima, Perú

Teléfono: (511) 961072186

Correo electrónico:montenegroidrogo@gmail.com

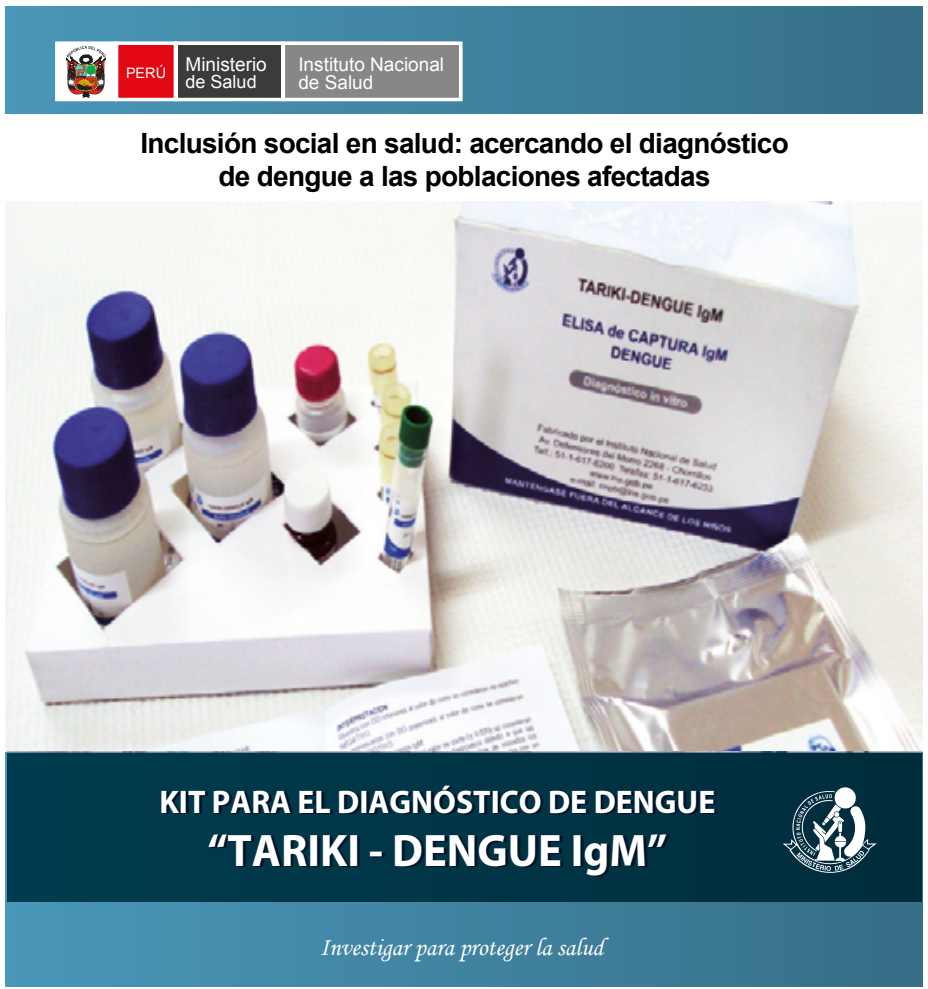

